\title{
Heparinoids: Structure, Biological Activities and Therapeutic Applications
}

\author{
Nur Sibel Gunay and Robert J. Linhardt* \\ Division of Medicinal and Natural Products Chemistry, College of Pharmacy and Department of Chemical and Biochemical Engineering, \\ University of lowa, lowa City, lowa, U.S.A.
}

Received: June 29, 1998; Revision accepted: December 20, 1998

\begin{abstract}
Heparin is an important polyanionic drug having a wide variety of different biological activities. Substantial research effort has focused on the preparation of improved heparins and heparin analogues that might exhibit higher specificity and decreased side effects. These heparin analogues or heparinoids include sulfated polysaccharides from plant and animal origin, synthetic derivatives of polysaccharides, and acidic oligosaccharides and their small synthetic analogues. The structure, biological activities and therapeutic potential of these heparinoids are discussed.
\end{abstract}

Key words: Heparin, heparinoid, glycosaminoglycan, sulfated polysaccharide, therapeutic applications.

\section{Introduction}

Heparin is a sulfated, linear polysaccharide that is derived from animal tissues and is widely used as a clinical anticoagulant (1). Despite its widespread use for over 60 years, the exact structure of heparin has not yet been established. Heparin is acidic (average negative charge of -100), polydisperse (chains ranging in molecular weight from 5,000 to $40,000)(1)$. It is comprised of a major (75-95\%) repeating disaccharide of $1 \rightarrow 4$ linked $\mathrm{N}$-sulfo-6-O-sulfo-D-glucosamine and 2-O-sulfoiduronic acid with minor sequences containing variable levels of $\mathrm{O}$ - and $\mathrm{N}$-sulfo groups and glucuronic acid (Fig. 1). Heparan sulfate (Fig. 1), is structurally similar to heparin and is found extracellularly, thus, most of the endogenous biological functions ascribed to heparin probably result from heparan sulfate (2).

Heparin is biosynthesized in mast cells as a proteoglycan (PG) (see Figs. 2 and 3), with approximately 10 glycosaminoglycan (GAG) side chains $(1,3)$. Heparin is stored intracellularly in mast cell granules and released on mast cell degranulation in an allergic response. It is extracted from mast cell rich tissue (i.e., lung, intestinal mucosa, etc.) in ton quantities and processed into pharmaceutical heparin. Heparin has a variety of biological activities many of which are of interest because of their potential therapeutic utility. The biological activities of heparin result from its binding to proteins modulating their

Planta Medica 65 (1999) 301 - 306

c) Georg Thieme Verlag Stuttgart · New York activities. These interactions are often very specific, e.g., heparin's anticoagulant activity primarily results from binding antithrombin III (ATIII, a serine protease inhibitor of thrombin and other coagulation proteases) at a discrete pentasaccharide sequence (Fig. 1) (4).

As a result of heparins multiplicity of biological activities and its importance as a major pharmaceutical, substantial research effort has been dedicated to the discovery of heparin analogues. This short review focuses on heparinoids, defined as heparin analogues from animal, plant and synthetic sources that mimic heparin's biological functions by interacting with heparin-binding proteins.

\section{Biological Activities of Polyanions}

\section{Structure and function of endogenous polyanions}

There are two major classes of endogenous polyanionic compounds found in animal tissues, the PGs/GAGs and the mucins $(1,5)$. These polyanions are found in pericellular locations, on the external surface of cell membranes, in the extracellular matrix occupying the spaces between cells and at the mucous membranes $(1,3)$. The primary function of the endogenous polyanions is believed to be cell-cell communication, as a barrier between tissues, cell adhesion, as a reservoir for growth factors and as a barrier protecting against pathogens $(1,3)$. Plant tissues also contain acidic polysaccharides. While most of these are polycarboxylated, i.e., alginate and pectin, some algal species contain sulfated polysaccharides, i.e., fucoidins and carrageenans (6). These polyanions serve a number of structural and protective roles in plants.

\section{Interaction of polyanions with proteins}

The biological activity of heparinoids is usually ascribed to their interaction with heparin-binding proteins. The large number of heparin-binding proteins can be classified into a small number of groups (Table $\mathbf{1}$ ). The term heparin-binding protein is somewhat of a misnomer since heparin is intracellular and has little if any opportunity to interact with extracellular proteins. A few proteins, such as chymase, found with heparin in mast cell granules, are true heparin-binding proteins, while most probably bind other endogenous, extracellular GAGs or mucins. 

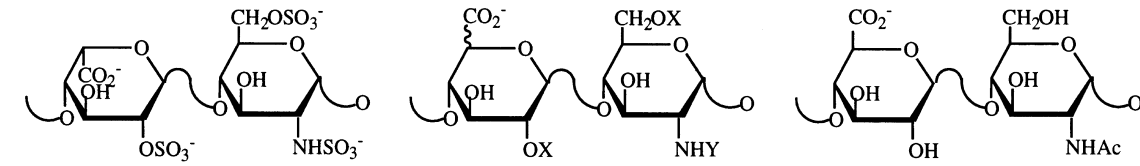

$\rightarrow-4)-\alpha-L-I d o A p 2 S(1 \rightarrow 4)-\alpha-D-G l c N p S 6 S(1 \rightarrow$ major disaccharide sequence
$\mathrm{X}=\mathrm{SO}_{3}{ }^{-}$or $\mathrm{H}, \mathrm{Y}=\mathrm{SO}_{3}{ }^{-}, \mathrm{CH}_{3} \mathrm{CO}$ or $\left.\mathrm{H} \rightarrow 4\right)-\beta-\mathrm{D}-\mathrm{GlcAp}(1 \rightarrow 4)-\alpha-\mathrm{D}-\mathrm{GlcNpAc}(1 \rightarrow$ minor disaccharide sequence

major disaccharide sequence
Fig. 1 Structure of heparin and heparan sulfate GAGs. The major and minor sequences of heparin and heparan sulfate as well as the ATIII binding site are shown.

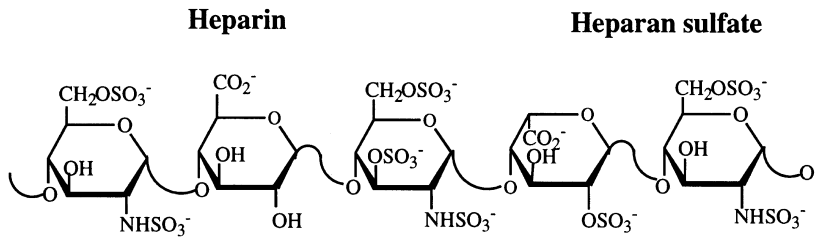

Antithrombin III binding sequence

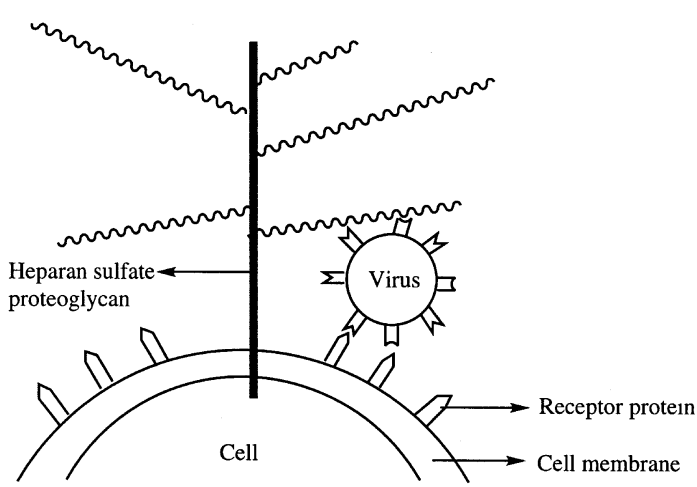

Fig. 2 Proposed mechanism of viral localization on an animal cell.

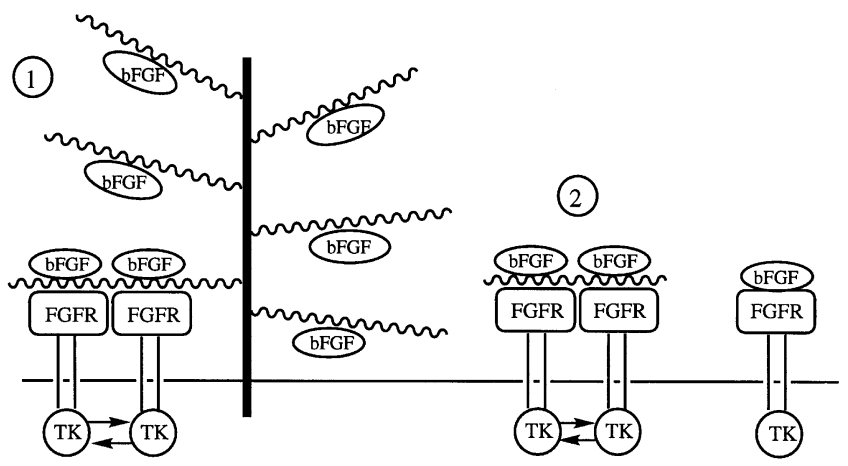

Fig. 3 Interaction of basic FGF on heparan sulfate PG (1) and free GAG (2) induces FGFR dimerization, leading to tyrosine kinase (TK) activation and signal transduction.

The interaction of heparin-binding proteins with heparinoids usually involves both ionic and hydrogen bonding interactions (7). Arginine and lysine are positively charged under physiologic $\mathrm{pH}$ and capable of ion-pairing with the $\mathrm{O}$ - and $\mathrm{N}$ sulfo groups of heparinoids. Hydrogen bonding interactions can involve basic and other polar (i.e., Asn, Gln, Ser, etc.) amino acids. Typically, ionic and hydrogen bonding residues lie in a spatially close array on the surface or in a shallow binding pocket on the surface of the heparin-binding protein. While hydrophobic interactions have limited importance in heparin binding, substantial hydrophobic contributions to binding may result in the interaction of heparinoids having hydrophobic character (i.e., suramin) making these exquisitely potent agents.

The saccharide backbone present in heparin and many heparinoids present their anionic substituents (sulfate and carboxylate groups) in a complex spatial array owing to the high level of chirality, different regioisomers, multiple conformers and secondary structural features of the carbohydrate backbone. Moreover, the flexibility of the saccharide backbone permits re-orientation of these charge groups during binding facilitating these interactions. Heparin (and to a lesser extent heparan and dermatan sulfate) contain a flexible L-iduronic acid that in many cases is essential for binding. Occassionally, heparin-protein interactions (i.e., selectin, annexins) even require a divalent metal, i.e., $\mathrm{Ca}^{2+}$ for binding.

Table 1 Heparin binding proteins (1).

\begin{tabular}{ll}
\hline Enzymes & Selectins \\
Lipolytic enzymes & \\
Kinases & Extracellular Matrix Proteins \\
Phosphatases & Collagens \\
Carbohydrate hydrolases, eliminases, & Fibronectin \\
transferases & Laminin \\
Proteases and esterases & Thrombospondin \\
Nucleases, polymerases, topoisomerases & Vitronectin \\
Other enzymes, oxidases, synthetases, & Receptor Proteins \\
dismutases & Steroid receptors \\
Enzyme Inhibitors & Growth factor receptors \\
Serine protease inhibitors (serpins) & Channel proteins \\
Lipoproteins & Viral Coat Proteins \\
Low and very low density lipoproteins & HIV \\
Apolipoproteins & HSV \\
Growth Factors & Dengue \\
Fibroblast growth factors & Nuclear Proteins \\
Epidermal growth factors & Histones \\
Hepatocyte growth factors & Transcription factors \\
Platelet-derived growth factors & Others \\
Transforming growth factors & Other Proteins \\
Vascular endothelial growth factors & Prion proteins \\
Chemokines & Amyloid proteins \\
& Fibrin \\
\hline
\end{tabular}




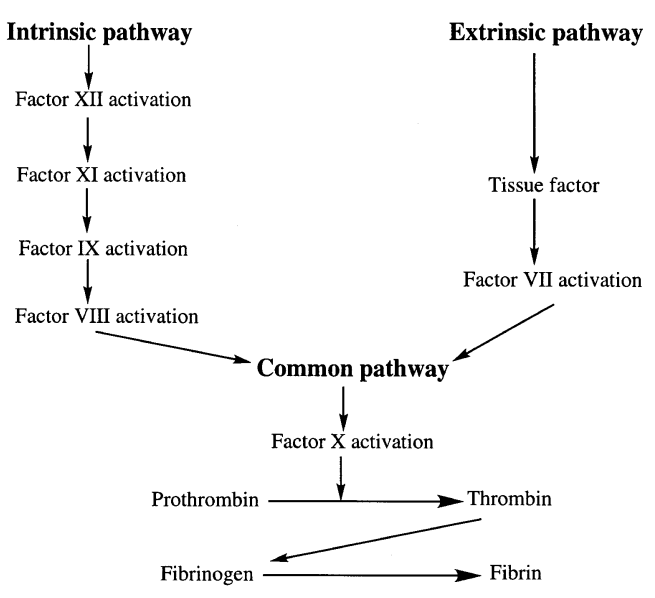

Fig. 4 Blood coagulation cascade.

\section{Inhibition of enzymatic processes}

Many heparin-binding proteins are enzymes or enzyme inhibitors. For example, proteases in the coagulation cascade (Fig. 4), such as factors IIa, IXa, Xa, XIa, XIIa, and the factor VIIIa, are heparin-binding proteins. Inhibitors of the coagulation cascade, such as ATIII and heparin cofactor II (HCII) as well as the enzymes and inhibitors of other biochemical cascades, such as the complement pathway, are also heparinbinding proteins $(1,8)$. Other enzymes, such as lipolytic enzymes, contain allosteric sites that are important for both the localization and the control of their activities (9).

\section{Importance in the maintenance of tissues and cell-cell interaction}

Endogenous extracellular GAGs lie on the surface of cells and are found in the extracellular matrix between cells that demarcate one tissue from another. These GAGs bind adhesion proteins (i.e., collagens, fibronectin, laminin, etc.) and are important in maintaining cell-cell contact. In addition to maintaining normal physiologic contact between cells within tissue and between tissues, pathologic processes of tumor angiogenesis and wound healing involve GAGs. Moreover, pathogens, such as parasites, bacteria and viruses, (Fig. 2) utilize GAGs to localize to the surface of animal cells in infection (10).

\section{Importance at receptors and signal transduction}

Important research is aimed at understanding GAG interaction with growth factors and their cellular receptors, the tyrosine kinases (11). The fibroblast growth factors (FGFs) are among the most extensively studied of the heparin-binding growth factors. Cell surface heparan sulfate PG serves as a reservoir for these growth factors. The currently held mechanism of action for FGF (Fig. 3) involves binding to a GAG sidechain of heparan sulfate PG, followed by FGF dimerization. Presentation of dimerized FGF to its tyrosine kinase receptor (also a heparin-binding protein) results in receptor dimerization and autophosphorylation. This reaction begins an intracellular biochemical cascade resulting in cell replication (or growth). A single heparin chain, an oligosaccharide of suffi- cient length (i.e., dodeca- or tetradecasaccharide (11)), or a heparinoid can often substitute for the GAG and stimulate growth.

\section{Involvement in nuclear events}

A number of nuclear proteins, i.e., histones, transcription factors, topoisomerase, (Table 1) bind heparin. Although heparan sulfate has been reported to be translocated to the nucleus, it is unclear what role if any endogenous GAGs might play in controlling nuclear events (3). One role would involve the GAG based transport of growth factors into the nucleus resulting in transcription and cell replication.

\section{Heparinoids}

\section{Naturally occurring sulfated polysaccharides}

From animal sources: A major group of sulfated polysaccharides found in animal tissues are the GAGs (Fig. 5). Dermatan sulfate (chondroitin sulfate B) is found in a wide variety of tissues in virtually all animals, and is a ( $1 \rightarrow 3,4$ linked) copolymer of $\mathrm{N}$-acetyl-D-galactosamine, L-iduronic acid and Dglucuronic acid with $\mathrm{O}$-sulfo groups commonly found on the 4-position of the $\mathrm{N}$-acetyl-D-galactosamine residues. Dermatan sulfate has been used clinically as an antithrombotic agent (12). Chondroitin sulfate is composed of repeating $(1 \rightarrow$ 3,4 linked) disaccharide units of D-glucuronic acid and $\mathrm{N}$ acetyl-D-galactosamine, with the $O$-sulfo groups at either the 4-position (chondroitin sulfate A) or 6-position (chondroitin sulfate $\mathrm{C}$ ) of the $\mathrm{N}$-acetyl-D-galactosamine. Sequence domains having increased (chondroitin sulfates D and E) and reduced (chondroitin) 0 -sulfo group content have been reported (1). Chondroitin sulfates are often considered to have reduced biological activity due to the absence of the flexible L-iduronic acid $(1,3)$. Acharan sulfate is composed of a simple disaccharide repeating unit of $1 \rightarrow 4$ linked $N$-acetyl-D-glucosamine and 2-O-sulfo-L-iduronic acid and is isolated from the giant African snail, Achatina fulica. The natural function of this molecule in snail, while still unclear, may be as an antidesiccant, a metal chelator, an anti-infective or a locomotive (slime) agent. Acharan sulfate has recently been shown to inhibit heparin's FGF-2 mitogenic activity in vitro (13). Keratan sulfate, the only GAG not to possess a uronic acid residue, is composed of repeating disaccharide units of galactose and $\mathrm{N}$-acetylglucosamine (14). The glucosamine and galactose residue are commonly 6 - $O$-sulfonated. Keratan sulfate, an important component of cartilage and the cornea, is involved in diseases associated with these tissues (14).

From plant sources: Fucoidin, a homopolymer of sulfated Lfucose, is isolated from plant tissue (6). Fucoidin is known to interfere with the function of P- and L-selectin, to inhibit LPSinduced leukocyte rolling and adhesion. Fucoidin also has been reported to have antithrombotic and anti-infective activities (15). Carrageenans are obtained commercially by aqueous extraction of certain species of red seaweeds in the Rhodophyceae class. They consist of sulfate esters of $(1 \rightarrow 3,4$ linked) D-galactose and 3,6-anhydro-D-galactose copolymers (6). Carrageenans provide structural support for the spatial arrangement of plants and are widely used in food, pharmaceutical and industrial applications (16). 


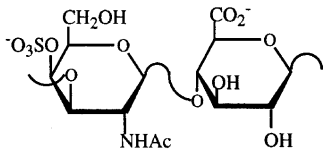

Chondroitin-4- $O$-sulfate

(Chondroitin sulfate A)

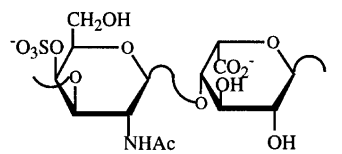

Dermatan sulfate

(Chondroitin sulfate B)

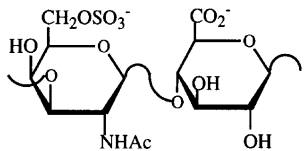

Chondroitin-6- $\boldsymbol{O}$-sulfate

(Chondroitin sulfate $\mathrm{C}$ )

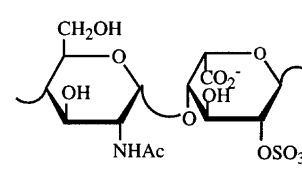

Acharan sulfate

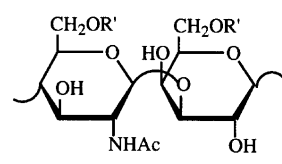

$\mathrm{R}=\mathrm{H}$ or $\mathrm{SO}_{3}{ }^{-}$ Keratan sulfate
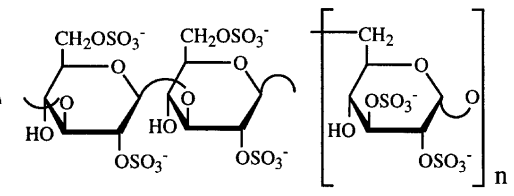

Laminarin sulfate
Fig. 5 The structures of the major disaccharide units of chondroitin sulfate, dermatan sulfate, acharan sulfate, keratan sulfate and laminarin sulfate as well as representative saccharide units of dextran sulfate, chitosan sulfate, pentosan sulfate and per$O$-sulfated hyaluronic acid (Ac is acetate and $\mathrm{n}$ is the degree of polymerization).

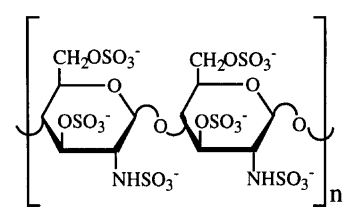

Chitosan sulfate

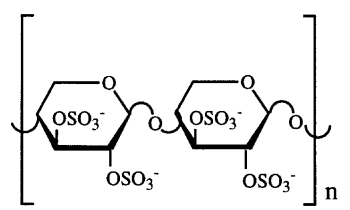

Pentosan sulfate

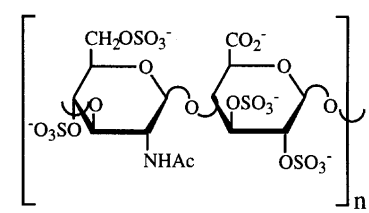

Per-O-sulfated hyaluronic acid
From microbial sources: Sulfated polysaccharides are rarely found as microbial products. This may be due to the absence of a highly compartmentalized cellular structure found in eucaryotes that is involved in the biosynthesis of sulfated polysaccharides (17). Acidic polysaccharides found in plants including hyaluronan, alginic acid and the K5 polysaccharide (from E.coli) have been used as precursors that when sulfonated afford important classes of heparinoids.

\section{Sulfation of neutral polysaccharides}

Chitin, poly(2-acetamido-2-deoxy-D-glucopyranose) is the major organic component of the exoskeleton found in insects, crabs, etc. $(1,6)$. Chitin can be de-N-acetylated to prepare chitosan (Fig. 5), which on chemical sulfonation has structural similarity to heparin and inhibits thrombin activity through ATIII (1). Pentosan, a ( $1 \rightarrow 4$ linked) xylopyranose with a single laterally positioned $4-0$-methyl- $\alpha$-D-glucuronic acid is extracted from the bark of the beech tree, Fagus sylvantica (1, 6). On sulfonation (Fig. 5), it has been shown to be an anticoagulant with one-tenth of heparin's activity on a weight basis when fully sulfated by chemical methods (1). Chemically sulfonated dextran, ( $1 \rightarrow 3,4$ linked) branched glucan polymer (Fig.5), has low anticoagulant activity with high lipoprotein releasing activity (1) and is used as an antiatherosclerotic in Japan (1). Dextran sulfate also is an inhibitor of human immunodeficiency virus (HIV) binding to T-lymphocytes but because of its low oral bioavailability it has not been used therapeutically (1). Laminarin, isolated from the cell wall of seaweed and composed of ( $1 \rightarrow 3$ linked) glucose residues is chemically sulfonated to prepare laminarin sulfate (Fig. 5) which is effective in the prevention and treatment of ischemic cerebrovascular disease (18). Treatment of capsular K5 polysaccharide from E.coli with mild acid (to remove branches) affords a ( $1 \rightarrow 4$ linked) copolymer of $N$-acetyl-D-glucosamine and D-glucuronic acid. De- $N$-acetylation, $\mathrm{N}$ - and $\mathrm{O}$-sulfonation (and potentially enzymatic C-5 epimerization) affords a heparin (or heparan sulfate)-like polysaccharide. The biological acitvities of $\mathrm{K} 5$ derivatives resulting from some or all of these transformations have been studied (17).

Hyaluronic acid is a very high molecular weight unsulfated GAG (1). Chemical $O$-sulfonation of this polysaccharide (Fig.5) affords a polysaccharide displaying anticoagulant activity (16). Complete de- $N$-acetylation of chondroitin, dermatan and acharan sulfates and $\mathrm{N}$-sulfonation affords unnatural products with interesting biological properties $(19,20)$. Heparin, heparan sulfate, dermatan sulfate and chondroitin sulfate all contain hydroxy groups that can also be partially or fully 0 -sulfonated (20).

\section{Sulfated oligosaccharides}

Controlled chemical and enzymatic depolymerization of GAGs afford sulfated low molecular weight (LMW) polysaccharides and sulfated oligosaccharides. Depolymerization methods include oxidative cleavage with peroxide, nitrous acid and base ( $\beta$-elimination) and enzymatic cleavage with heparinase (1). LMW heparins are polydisperse mixtures (average molecular weight 4,000 to 8,000 ) that are used as anticoagulant/ antithrombotic drugs. The major advantages of these agents is their enhanced subcutaneous bioavailability (compared to heparin) and their elevated ratio of anti-factor Xa:anti-factor Ila activity (1). Controlled enzymatic depolymerization of heparin with heparinase has also led to the preparation and purification (to homogeneity) of heparin oligosaccharides (1). These have been extremely useful for studying the multiple biological activities of heparin by dissecting apart the specific binding sites within the heparin polysaccharide for different heparin binding proteins. For example, a hexasaccharide containing the sequence within heparin that binds FGF-2 (11) has been prepared by this approach. Structurally defined, homogenous, heparin-derived oligosaccharides offer the opportunity to regulate specific biological pathways. Sucrose 
can be completely $O$-sulfonated to obtain sucrose octasulfate (Fig. 6), which forms an insoluble aluminum salt called Sucralfate $\mathrm{TM}^{\mathrm{TM}}$ and is used for the treatment of ulcers (1). Synthetic, sulfated lactobionic acids can be easily prepared from lactose and are very active in potentiating the HCII mediated inhibition of thrombin (1).

The first total synthesis of a heparin pentasaccharide ATIII binding site in 1984 (4) required multiple synthetic and separation steps resulting in a very low overall yield. Subsequent syntheses have reduced the required steps and increased the yield leading to its development as an anticoagulant drug.

\section{Synthetic sulfated and sulfonated polymers}

Poly(vinyl sulfate) and poly(anethole sulfonate) exhibit anticoagulant activity (Fig. 7) (1) that has been exploited in vitro to prevent coagulation of collected blood (1). A biphenyl disulphonic acid urea co-polymer shows potent anti-HIV activity (21). Synthetic, non carbohydrate-derived heparinoids are generally toxic because of their long half-life resulting from their failure to be cleared either through filtration or metabolism.

\section{Small sulfated and sulfonated compounds}

Suramin (Fig. 6), one of the oldest heparinoids, shows activity as an anthelmintic, an antiprotozoal, an antineoplastic and an antiviral agent (1). Despite its potent activities, suramin has a long half-life and exhibits a wide range of toxic side effects. Naphthalenesulfonates (Fig. 6) show potent anti-HIV activity (1), but limited toxicological data are currently available on these compounds. A series of simple aliphatic disulfates (Fig. 6) and disulfonates have been synthesized and tested for their ability to arrest amyloidosis in vivo as potential agents for the treatment of Alzheimer's disease (22).

\section{Potential Therapeutic Applications of Polyanions}

The only currently approved therapeutic applications of heparinoids are as anticoagulant/antithrombotic agents and for the preparation of biomaterials used in medical devices
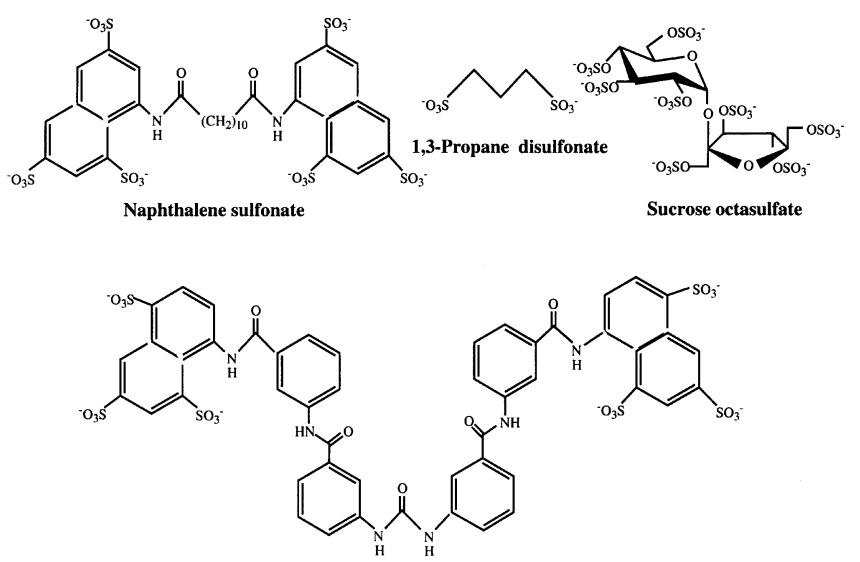

Suramin

Fig. 6 Structures of synthetic and semisynthetic heparinoids. (i.e., catheters, hollow fiber artificial kidneys, blood oxygenators, stents) (23). There are additional therapeutic areas that are under extensive investigation (Table 2 ). Three of these areas are described below in detail.

\section{Regulation of enzymatic processes}

Since this is the major current application for heparin as an anticoagulant/antithrombotic, it is not surprising that much of the research has targeted this type of activity. The extensive evaluation of heparinoids as anticoagulant/antithrombotic agents has met with limited success (1). Recently heparinoids have been evaluated as regulators of the complement cascade and proteases, such as elastase, for the treatment of lung disorders such as hereditary angioedema (8) and asthma (24).

\section{Regulation of growth factors and signal transduction}

The extensive studies aimed at understanding the role of endogenous heparan sulfate in regulation of cellular growth has suggested the value of heparinoids as a potential new class of therapeutic agents. Such agents could be used in wound healing and in promoting angiogenesis in the regeneration of vessels following stroke (25). Alternatively, heparinoids might be useful in inhibiting tumor-promoted angiogenesis representing a new approach to the treatment of cancers (26).



Polyanethole sulfate



MDL 101028 Biphenyl disulphonic acid urea copolymer $(n=3-8)$

Fig. 7 Structures of synthetic polymers used as heparinoids ( $\mathrm{n}$ is degree of polymerization).

Table 2 Potential therapeutic applications for heparinoids.

\begin{tabular}{lll}
\hline Application & Status & Reference \\
\hline Anticoagulant/Antithrombotic & Currently used & 1 \\
Antiatherosclerotics & Clinical trials & 9 \\
Complement Inhibitors & Clinical trials & 8 \\
Anti-inflammatories & Animal studies & 24 \\
Anticancer Agents & Animal studies & 26 \\
Antiviral Agents & Animal studies & 27 \\
Antialzheimer Agents & Animal studies & 22 \\
Antiprion Agents & In vitro studies & 28 \\
Antiparasitic Agents & Animal studies & 10 \\
New Biomaterials & Currently used & 23 \\
\end{tabular}




\section{Anti-infectives}

It is clear that pathogens use endogenous GAGs to localize on the surface of cells and even to gain entrance into cells resulting in infection (10). The increasing number of reports of antibiotic resistant microorganisms suggest that new antiinfective mechanisms such as ones disrupting cellular localization of pathogens might be valuable. The dengue virus causing hemorrhagic fever in many tropical countries is an example of an infectious disease for which there is no vaccine available and no known specific treatment. Recently an oversulfated heparan sulfate chain was shown to be a receptor required for infectivity of this virus (27). Interestingly, a heparinoid, suramin can block such infection in vitro.

\section{Conclusions}

In conclusion, heparinoids represent therapeutically important target molecules. These heparinoids can be prepared from animal, plant and microbial sources and through chemical synthesis. Despite some successes in developing new heparinoid agents, substantial additional efforts are needed to expand both the types of molecules used as heparinoids and their different therapeutic applications.

\section{Acknowledgements}

This research was supported by the National Institutes of Health grants GM 38060 and HL 52622.

\section{References}

${ }^{1}$ Linhardt, R. J., Toida, T. (1997) Heparin Oligosaccharides: New Analogues Development and Applications, in: Carbohydrate in Drug Designing, (Zbigniew, J. W., Karl, A. N., eds.), Marcel Dekker, Inc., New York, pp. 277-341.

2 Stringer, S. E., Gallagher, J. T. (1997) Int. J. Biochem. Cell. Biol. 29, $709-714$

${ }^{3}$ Conrad, H. E. (ed.) (1998) Heparin-Binding Proteins, Academic Press, California.

4 Sinaÿ, P., Jacquinet, J. C., Petitou, M., Duchaussoy, P., Lederman, I., Choay, J., Torri, G. (1984) Carbohydr. Res. 132, C5 - C9.

5 Devine, P. L., McKenzie, F. C. (1992) BioEssays 14, 619-625.

${ }^{6}$ Kennedy, J. F., White, C. A. (eds.) (1983) Bioactive Carbohydrates, Ellis Harwood, Ltd., New York.

${ }^{7}$ Hileman, R. E., Fromm, J. R., Weiler, J. M., Linhardt, R. J. (1998) BioEssays 20, 156 - 167.

${ }^{8}$ Edens, R. E., Linhardt, R. J., Weiler, J. M. (1993) Compl. Prof. 1, 96 120.

9 Engelberg, H. (1996) Pharmacol. Rev. 48, 327-352.

10 Sawitzky, D. (1996) Med. Microbiol. Immunol. 184, 155-161.

11 Faham, S., Linhardt, R. J., Rees, D. C. (1998) Curr. Opin. Struct. Biol. $8,578-586$.

12 Linhardt, R. J., Hileman, R. E. (1995) Gen. Pharmacol. 26, 443 - 451.

13 Wang, H., Toida, T., Kim, Y. S., Capila, I., Hileman, R. E., Bernfield, M., Linhardt, R. J. (1997) Biochem. Biophys. Res. Commun. 235, 369-373; Erratum 242, 248, 1998.

14 Greiling, H., Scott, J. (eds.) (1989) Keratan Sulfate Chemistry, Biology, Chemical Pathology, T. J. Press Ltd., Great Britain.

15 Shimaoka, M., Ikeda, M., Iida, T., Taenaka, N., Yoshiya, I., Honda, T. (1996) Am. J. Respir. Care Med. 153, 307 - 311.

16 Weiner, M. L. (1991) Agents and Actions 32, 46-51.

17 Razi, N., Feyzi, E., Bjork, I., Naggi, A., Casu, B., Lindahl, U. (1995) Biochem. J. 309, 465-472.
18 Miao, H.-Q., Ishai-Michaeli, R., Peretz, T., Vlodavsky, I. (1995) J. Cell. Physiol. 164, 482-490.

19 Maruyama, T., Toida, T., Imanari, T., Yu, G., Linhardt, R. J. (1998) Carbohydr. Res. 306, 35- 43.

${ }^{20}$ Nadkarni, V. D., Toida, T., Van Gorp, C. L., Schubert, T. L., Weiler, J. M., Hanse, K. P., Caldwell, E. E. O., Linhardt, R. J. (1996) Carbohydr. Res. 290, 87-96.

21 Taylor, D. L., Brennan, T. M., Bridges, C. G., Mullins, M. J., Tyms, A. S., Jackson, R., Cardin, A. D. (1995) Antiviral Research 28, 159173.

22 Kisilevsky, R., Lemieux, L. J., Fraser, P. E., Kang, X., Hultin, P. G., Szarek, W. A. (1995) Nature Medicine 1,143-148.

${ }^{23}$ Fareed, J. (1996) Sem. Thromb. Hemostas. 22, 3 - 8 .

${ }^{24}$ Fath, M. A., Wu, X., Hileman, R. E., Linhardt, R. J., Kashem, M. A., Nelson, R. M., Wright, C. D., Abraham, W. M. (1998) J. Biol. Chem. 273, 13563-13569.

25 Gordon, D. L., Linhardt, R. J., Adams, H. P. (1990) Clin. Neuropharmacol. 13, 522-543.

${ }^{26}$ Folkman, J., Langer, R., Linhardt, R. J., Haudenschild, C., Taylor, S. (1983) Science 221, 719-725.

27 Chen, Y., Maguire, T., Hileman, R. E., Fromm, J. R., Esko, J. D., Linhardt, R. J., Marks, R. M. (1997) Nature Med. 3, 866 - 871.

28 Shyng, S-L., Lehmann, S., Moulder, K. L., Harris, D. A. (1995) J. Biol. Chem. 270, 30221 - 30229.

\section{Dr. Robert J. Linhardt}

Division of Medicinal and Natural Products Chemistry College of Pharmacy and Department of Chemical and Biochemical Engineering PHAR S342

University of Iowa

Iowa City

Iowa 52242

U.S.A.

E-mail: robert-linhardt@uiowa.edu

Fax: +1319-335-6634 